\title{
Mechanism of Endotracheal Tube Movement with Change of Head Position in the Neonate
}

\author{
S. M. Donn and L. R. Kuhns \\ Departments of Pediatrics and Communicable Diseases, and Division of Pediatric Radiology, C. S. Mott Children's Hospital, University of \\ Michigan Medical Center, Ann Arbor, Michigan, USA
}

\begin{abstract}
The mechanism of alteration of endotracheal tube position with movement of the head and neck in the neonate was studied in a term newborn cadaver. The infant was intubated and serial radiographs were obtained with the head and neck in different positions. We propose that the skull acts as a lever arm from the anterior end of the maxilla to the first cervical vertebra. The fulcrum for movement of this lever arm is the upper cervical spine. Movement of the endotracheal tube in the trachea is directed by the maxillocervical lever arm when the skull and upper cervical spine are flexed, extended, or rotated.
\end{abstract}

Key words: Endotracheal tube - Neonate - Radiography

Several investigators have reported alteration of endotracheal tube position with head movement and its potential deleterious clinical effects in both adults and children [1-6]. A theoretical explanation for the mechanism of tube movement has been proposed for adults [2], although this hypothesis was not based on experimental data. To our knowledge, the mechanism of tube movement has not been studied in the neonate. We report herein the results of an investigation into the mechanism of endotracheal tube movement in the neonate.

\section{Methods and Materials}

The study was performed post-mortem on a term newborn, 24 hours after he expired on the second day of life. The infant had a meningomyelocele and Arnold-Chiari malformation, but subse- quent autopsy revealed no additional anomalies of the head and neck or airway. There was no rigor mortis. Informed consent had been obtained from the parents, who had released the body for anatomical studies.

The infant was intubated orally with a $3.5 \mathrm{~mm}$ constant diameter polyvinyl chloride endotracheal tube with a radiopaque marker at its tip. The tube was inserted to its $9 \mathrm{~cm}$ marker, and with the head in the neutral position the tip was located radiographically at the level of the thoracic inlet. The tube was (as in clinical use) secured with tape, and a stainless steel safety pin was placed through the tube at the level of the upper lip to serve as a reference marker and to prevent inadvertent movement of the tube in relation to the maxilla during changes of head position.

Lateral radiographs of the head and neck were obtained with the infant's head in the flexed, neutral, and extended positions, and with the head rotated laterally. Antero-posterior radiographs were obtained with the head in neutral and rotated positions.

\section{Results}

Figures 1 and 2 show the change of the endotracheal tube position with movement of the head and neck. These results are in agreement with previous studies [2-6]; the tube tip moves caudad (towards the carina) with flexion, and cephalad (towards the glottic opening) with extension. Lateral rotation causes the tube tip to move cephalad as well. There was no movement of the reference marker in relation to the maxilla, indicating that the tube remained stationary at its site of fixation to the mouth.

\section{Discussion}

Figure 3 represents a schematic diagram of our proposed mechanism for the movement of the endotracheal tube tip with change in head and neck position. In our neonate flexion and extension of the cervical spine occurred primarily through the first four cervical vertebrae $\left(\mathrm{C}_{1}-\mathrm{C}_{4}\right.$ in the diagram) with the 

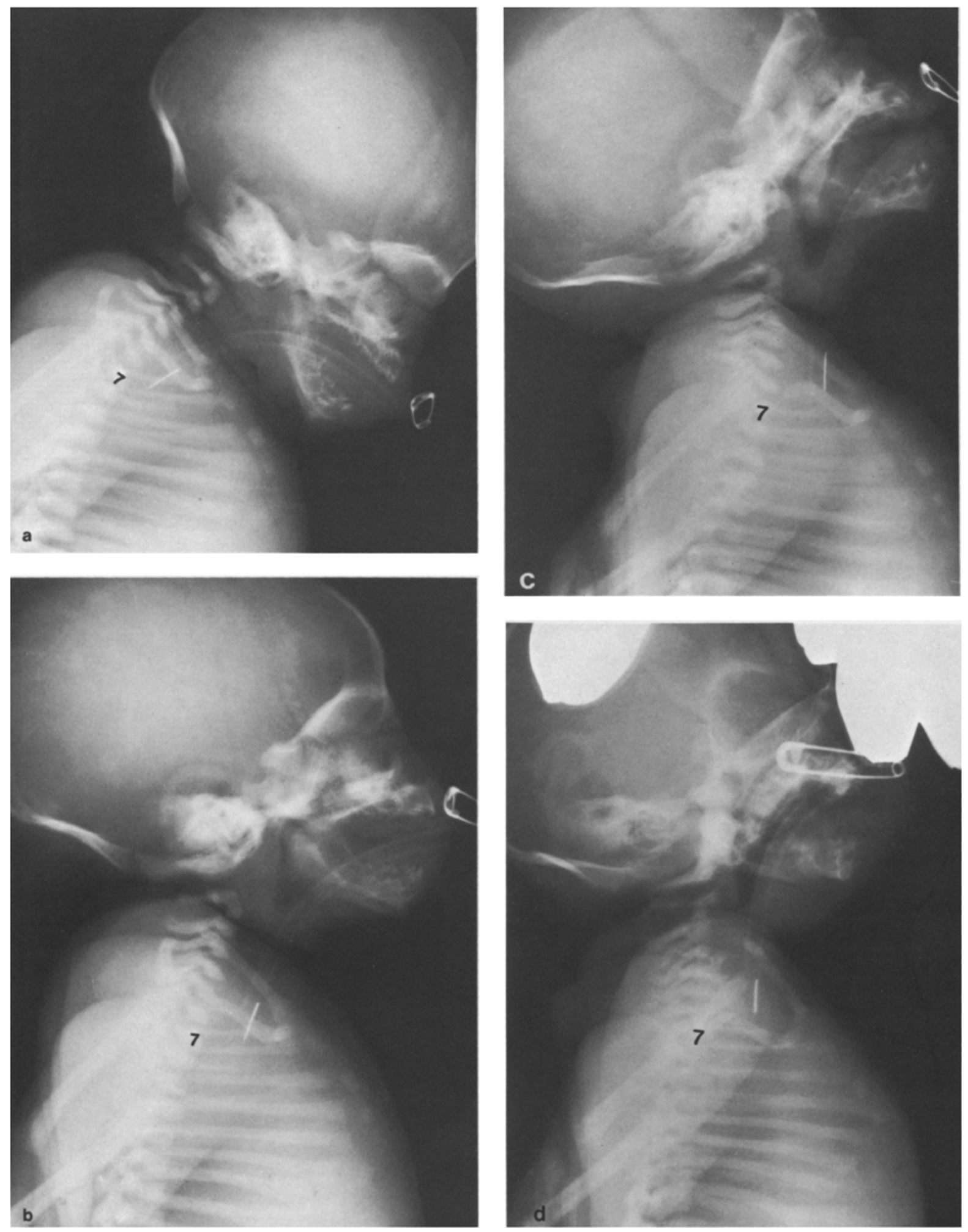

Fig. 1a-d. Lateral radiographs of intubated neonate cadaver. Body of seventh cervical vertebra is marked 7. a Head and neck in neutral position: Endotracheal tube tip is at level of $\mathrm{C}_{6}-\mathrm{C}_{7}$ interspace. b Head and neck in flexed position: Endotracheal tube tip is at $\mathrm{C}_{7}$. $\mathbf{c}$ Head and neck in extended position: Endotracheal tube tip is at $\mathrm{C}_{6}$. $\mathbf{d}$ Chin in neutral position, head rotated laterally: Endotracheal tube tip is at $\mathrm{C}_{6}$ 

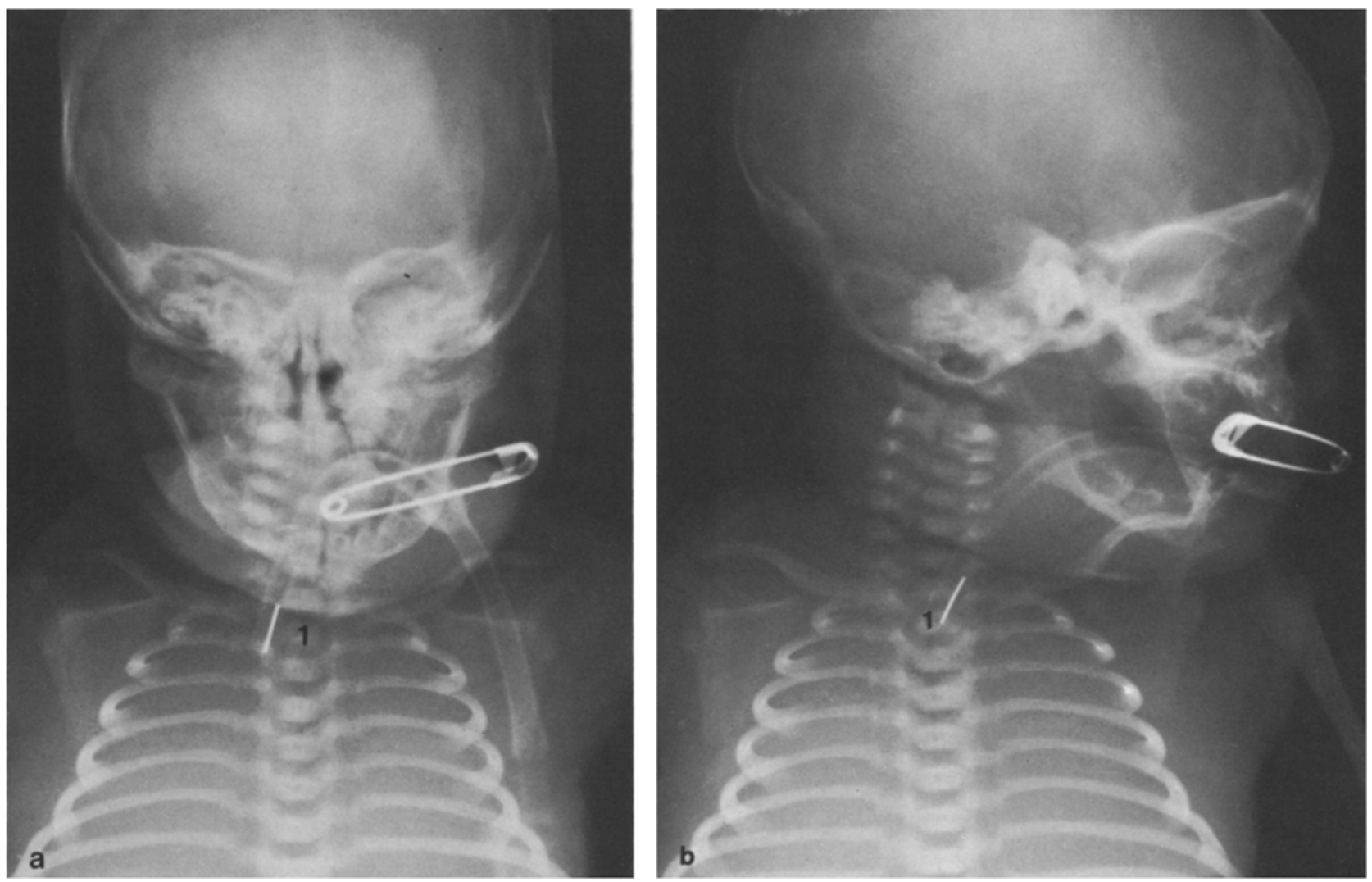

Fig. 2 a and b. Antero-posterior radiographs of same neonate. Body of first thoracic vertebra is marked 1 . a Head and neck in neutral position: Endotracheal tube tip is at $T_{1}-T_{2}$ interspace. $\mathbf{b}$ Head and neck in lateral rotation: Endotracheal tube tip is at $T_{1}$

lower cervical spine $\left(\mathrm{C}_{4}-\mathrm{C}_{7}\right)$ relatively fixed. The length of the endotracheal tube is constant, as is its fixed position at the mouth. The skull, from the anterior tip of the maxilla to the first cervical vertebra, acts as a lever arm, moving about a fulcrum centered at the upper cervical spine. Movement of the tube tip occurs because the functional lever arm is applied to the endotracheal tube. When the neck is flexed the lever arm is rotated inferiorly, which forces the endotracheal tube tip to assume a more caudal position. When the neck is extended the opposite effect occurs, drawing the tip cephalad. Lateral head movement introduces a rotary component and draws the tip cephalad; the lever arm serves as a radius describing an arc whose center is the atlas. This rotary motion is directed away from the carina, pulling the tube tip with it.

This proposed mechanism of tube movement is based on direct radiographic observations of an intubated neonate. Conrady et al. [2] proposed a mechanism for tube tip movement during flexion and extension of the adult neck. They conceptualized the endotracheal tube and the vertebral column as concentric circles. During flexion the tube is the inner circle, and the vertebral column is the outer circle.

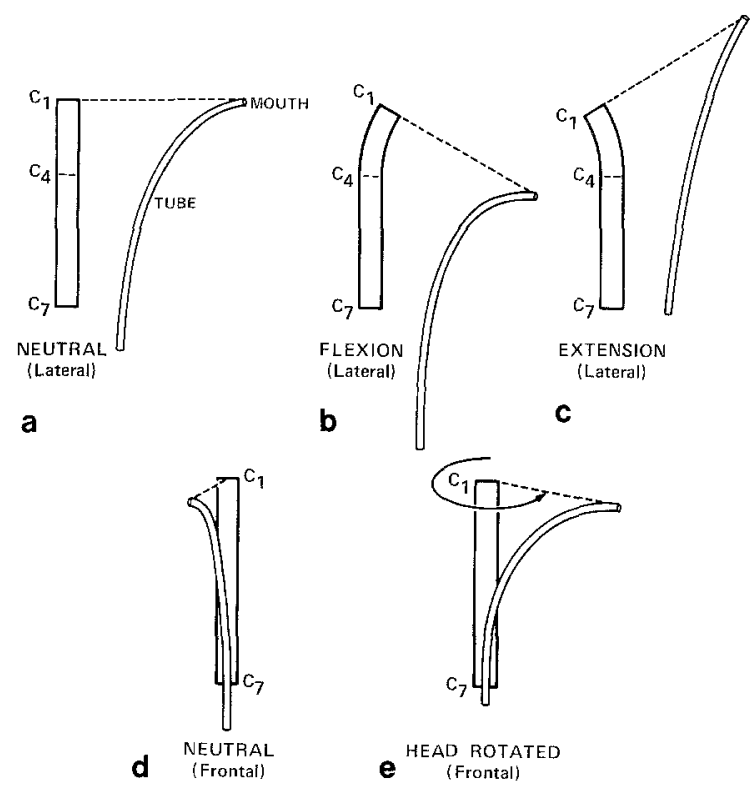

Fig. 3a-e. Schematic diagram of proposed mechanism. a Neutral position, lateral view. b Flexion, lateral view. Note lever arm $\left(\mathrm{C}_{1}{ }^{-}\right.$ mouth) has rotated inferiorly around $\mathrm{C}_{1}-\mathrm{C}_{4}$ fulcrum pushing tube tip caudad. c Extension, lateral view. Lever arm has rotated superiorly, pulling tube tip cephalad. d Neutral position, frontal view. e Lateral rotation, frontal view. Lever arm has rotated laterally describing an are about $\mathrm{C}_{1}$, drawing tube tip away from carina 
During extension, the situation is reversed. The circumference of the outer circle is always greater than that of the inner, and because the length and fixation of the tube are constant, when the tube is the inner circle (flexion) it moves toward the carina, and when it is the outer circle (extension) it moves away from the carina. Their hypothesis is based on lateral spine radiographs of an adult who was not intubated; flexion and extension of the neck involved movement of all the cervical vertebral bodies in their example. This was not the case in our study, where flexion and extension of the head and neck in our neonate resulted in movement of the upper cervical vertebrae only. Therefore, the mechanism described in adults may not apply to neonates. Conrady et al. proposed no mechanism for tube movement with lateral rotation of the neck in adults. Our lever arm-fulcrum concept provides an explanation for endotracheal tube movement with flexion, extension, and rotation of the head and neck, and we feel it is more applicable to neonates than the mechanism proposed by Conrady.

These results indicate that the mandible should be included in chest radiographs that are obtained for evaluation of endotracheal tube placement. If the neck is flexed in an anteroposterior radiograph as the technologist slides the cassette beneath the neonate, the tube tip will be apparently too low in the trachea. When the technologist removes the cassette and extends the neck, the subsequent withdrawal of the endotracheal tube by the neonatologist may result in inadvertent extubation. Conversely, if the radiograph is obtained with the neck extended, the tube may be apparently too high in the trachea. Subsequent advancement of the tube with the neck in neutral position may result in inadvertent intubation of the right main bronchus.

Acknowledgements. The authors wish to thank Drs. D. W. Roloff and $\mathrm{J}$. F. Holt for their assistance in preparing this manuscript.

\section{References}

1. Bednarek, F. J., Kuhns, L. R.: Endotracheal tube placement in infants determined by suprasternal palpation: A new technique. Pediatrics 56, 224 (1975)

2. Conrady, P., Goodman, L., Laing, F., et al.: Alteration of endotracheal tube position - flexion and extension of the neck. Crit. Care Med. 4, 8 (1976)

3. Goodman, L. R., Conrady, P. A., Laing, F., et al.: Radiographic evaluation of endotracheal tube position. A. J. R. 127, 433 (1976)

4. Heinonen, J., Takki, S., Tammisto, T.: Effect of the Trendelenburg tilt and other procedures on the position of endotracheal tubes. Lancet 1969 I, 850

5. Kuhns, L. R., Poznanski, A. K.: Endotracheal tube position in the infant. J. Pediatr. 78, 991 (1971)

6. Todres, I. D., deBras, F., Kramer, S. S., et al.: Endotracheal tube displacement in the newborn infant. J. Pediatr. 89, 126 (1976)

Date of final acceptance: July 25, 1979

Lawrence R. Kuhns, M. D.

Division of Pediatric Radiology

C. S. Mott Children's Hospital

University of Michigan Medical Center

Ann Arbor, MI 48109

USA 